\title{
THE
}

4-15-1996

\section{Dynamics of Quantum Spin Systems in Dimer and Valence-Bond- Solid Ground States Stabilized by Competing Interactions}

Yongmin $\mathrm{Yu}$

University of Rhode Island

Gerhard Müller

University of Rhode Island, gmuller@uri.edu

Follow this and additional works at: https://digitalcommons.uri.edu/phys_facpubs

Terms of Use

All rights reserved under copyright.

\section{Citation/Publisher Attribution}

Yongmin Yu and Gerhard Müller. Dynamics of quantum spin systems in dimer and valence-bond-solid ground states stabilized by competing interactions. J. Appl. Phys. 79 (1996), 4629-4631.

Available at: http://dx.doi.org/10.1063/1.361686

This Article is brought to you for free and open access by the Physics at DigitalCommons@URI. It has been accepted for inclusion in Physics Faculty Publications by an authorized administrator of DigitalCommons@URI. For more information, please contact digitalcommons-group@uri.edu. 


\title{
Dynamics of quantum spin systems in dimer and valence-bond-solid ground states stabilized by competing interactions
}

\author{
Yongmin Yu and Gerhard Müller \\ Department of Physics, The University of Rhode Island, Kingston, Rhode Island 02881-0817
}

\begin{abstract}
For special coupling ratios, the one-dimensional (1D) $s=1 / 2$ Heisenberg model with antiferromagnetic nearest and next-nearest neighbor interactions has a pure dimer ground state, and the 1D $s=1$ Heisenberg model with antiferromagnetic bilinear and biquadratic interactions has an exact valence-bond-solid ground state. The recursion method is used to calculate the $T=0$ spin dynamic structure factor for both models and, for the $s=1 / 2$ model, also the dimer dynamic structure factor. New results for line shapes and dynamically relevant dispersions are obtained. (C) 1996 American Institute of Physics. [S0021-8979(96)00208-7]
\end{abstract}

Correlated quantum fluctuations in the ground state are a generic feature of quantum many-body systems. They make it hard to take finite-size effects into account in computational studies of zero-temperature dynamical properties. Interestingly, there are several known cases, where a relatively simple-structured ground state is stabilized by competing terms in the microscopic Hamiltonian. This ground state may or may not be long-range ordered. The essential attribute is that its fluctuations are not correlated or only over a short distance on the lattice. This phenomenon typically occurs with no accompanying simplification in the excitation spectrum or any dynamical quantity. Nevertheless, any such situation provides an unsuspected window for dynamical studies which promise to be much less plagued with finite-size effects than is typically the case. The recursion method ${ }^{1}$ in combination with recently developed techniques of continued-fraction analysis ${ }^{2}$ is an ideal calculational tool for that purpose, the key property being that it extracts the dynamical information from the ground-state wave function.

In a previous paper we have reported the study of one such case, namely the one-dimensional (1D) spin-s $X Y Z$ model. ${ }^{3}$ In a magnetic field of particular strength, this model has a product ground state with spontaneous ferro- or antiferromagnetic long-range order perpendicular to the field. Here we present new results for two different models with simple-structured ground states.

The first model is the 1D $s=1 / 2$ Heisenberg antiferromagnet with competing nearest and next-nearest neighbor interactions,

$$
H=\sum_{l=1}^{N}\left\{J_{1} \mathbf{S}_{l} \cdot \mathbf{S}_{l+1}+J_{2} \mathbf{S}_{l} \cdot \mathbf{S}_{l+2}\right\}
$$

with an even number of spins and periodic boundary conditions. This system undergoes a $T=0$ phase transition at $J_{2} / J_{1} \simeq 0.25$ from a spin-fluid phase to a phase with spontaneous dimer long-range order. ${ }^{4}$ In the (critical) spin-fluid phase, the correlations of the quantum fluctuations are particularly strong. In the dimer phase, their continued presence, albeit much attenuated, manifests itself, for example, in the finite-size splitting of the ground-state doublet. The exception is the special coupling ratio $J_{2} / J_{1}=0.5$, where the pure dimer ground-state is realized. ${ }^{5,6}$ Here the ground-state energy per site is size-independent: $E_{0} / N=-\frac{3}{8} J_{1}$.
The two (translationally invariant) dimer ground-state wave functions can be expressed in terms of products of singlets formed by pairs of nearest neighbor spins:

$$
\left|\Phi_{ \pm}\right\rangle=\left[2 \pm(-1 / 2)^{(N-4) / 2}\right]^{-1 / 2}\left\{\left|\Phi_{1}\right\rangle \pm\left|\Phi_{2}\right\rangle\right\},
$$

where $\left|\Phi_{1}\right\rangle=[1,2][3,4] \cdots[\mathrm{N}-1, N],\left|\Phi_{2}\right\rangle=[2,3][4,5] \cdots[N, 1]$, $\times[l, l+1]=\{|\uparrow \downarrow\rangle-|\downarrow \uparrow\rangle\} / \sqrt{2}$.

The dimer order parameter, $D=N^{-1} \Sigma_{l}(-1)^{l} D_{l}, D_{l}$ $=S_{l}^{+} S_{l+1}^{-}+S_{l}^{-} S_{l+1}^{+}$, has a nonzero expectation value, $\langle D\rangle= \pm 1 / 2$ in the (non-orthogonal) symmetry-breaking states $\left|\Phi_{1}\right\rangle$ and $\left|\Phi_{2}\right\rangle$. The order-parameter correlation function in this case is not a two-spin correlation function, $\left(\left\langle S_{l}^{z} S_{l+n}^{z}\right\rangle=0\right.$ for $\left.|n|>1\right)$, but a four-spin correlation function: $\left\langle D_{l} D_{l+n}\right\rangle-\left\langle D_{l}\right\rangle\left\langle D_{l+n}\right\rangle=(-1)^{n} / 4$ for $n \neq 0$. Hence it will be instructive to compare the spin dynamic structure factor $S_{z z}(q, \omega)$ and the dimer dynamic structure factor $S_{D D}(q, \omega)$, i.e. the function

$$
S_{A A}(q, \omega)=\int_{-\infty}^{+\infty} d t e^{i \omega t}\left\langle A_{q}(t) A_{-q}\right\rangle,
$$

where $A_{q}$ stands for the spin fluctuation operator, $S_{q}^{z}$ $=N^{-1 / 2} \Sigma_{l} e^{i q l} S_{l}^{z}$ or the dimer fluctuation operator, $D_{q}$ $=N^{-1 / 2} \Sigma_{l} e^{i q l}\left[D_{l}-\left\langle D_{l}\right\rangle\right]$.

By means of the recursion method ${ }^{1}$ in combination with a strong-coupling continued-fraction analysis, ${ }^{2,7}$ we calculate the dynamically relevant excitation spectra and the spectralweight distributions of these two functions. The recursion algorithm in the present context is based on an orthogonal expansion of the wave function $\left|\Psi_{q}^{A}(t)\right\rangle=A_{q}(-t)|\Phi\rangle$. It produces (after some intermediate steps) a sequence of continued-fraction coefficients $\Delta_{1}^{A}(q), \Delta_{2}^{A}(q), \ldots$ for the relaxation function,

$$
c_{0}^{A A}(q, z)=\frac{1}{z+\frac{\Delta_{1}^{A}(q)}{z+\frac{\Delta_{2}^{A}(q)}{z+\ldots}},}
$$

which is the Laplace transform of the symmetrized correlation function $\Re\left\langle A_{q}(t) A_{-q}\right\rangle /\left\langle A_{q} A_{-q}\right\rangle$. The $T=0$ dynamic structure factor (3) is then obtained from (4) via

$$
S_{A A}(q, \omega)=4\left\langle A_{q} A_{-q}\right\rangle \Theta(\omega) \lim _{\varepsilon \rightarrow 0} \Re\left[c_{0}^{A A}(q, \varepsilon-i \omega)\right] .
$$




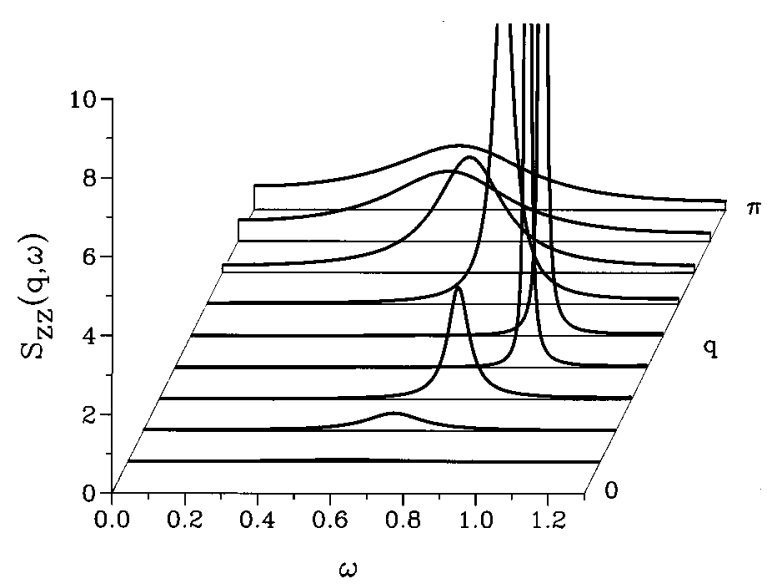

FIG. 1. Dynamic structure factor $S_{z z}(q, \omega)$ vs $\omega$ for $q=2 \pi l / N$, $l=0,1, \ldots, N / 2$ in the dimer ground state $\left|\Phi_{+}\right\rangle$of the Hamiltonian (1) at $J_{2}=0.5$ and $J_{1}=1$ with $N=18$, obtained via strong-coupling continuedfraction reconstruction based on the coefficients $\Delta_{1}, \ldots, \Delta_{9}$ and a Gaussian terminator as explained in Refs. 2 and 7.

The simple $N$-dependence of the dimer ground-state wave functions (2) offers the advantage that we can compute a significant number of $N$-independent coefficients $\Delta_{k}^{A}(q)$. These data are the input to the well-tested strong-coupling continued-fraction reconstruction. ${ }^{2,7}$

The results for the frequency-dependence of the dynamic structure factor $S_{z z}(q, \omega)$ at the wave numbers realized for $N=18$ are displayed in Fig. 1 . The set of curves is perfectly compatible with a function $S_{z z}(q, \omega)$ that varies smoothly in $q$ as well as in $\omega$. For every $q$-value we observe a single peak in the frequency range of interest. This peak is very broad at $q$ near zero or $\pi$. The width shrinks as $q$ approaches $\pi / 2$ from either side.

At $q=\pi / 2$ (not realized for $N=18$ ) the dynamically relevant excitation spectrum reduces to a single mode. The states $S_{\pi / 2}^{z}\left|\Phi_{ \pm}\right\rangle$are, in fact, known to be exact eigenstates of the system. 8,9 The dynamically relevant dispersion of $S_{z z}(q, \omega)$ is symmetric about $q=\pi / 2$, where it has a smooth maximum at frequency $\omega / J_{1}=1.0$. For $q=0$ and $q=\pi$ it has smooth minima at $\omega / J_{1} \simeq 0.5$.

On the basis of a variational calculation for the pure dimer state, Shastry and Sutherland ${ }^{6}$ obtained an excitation spectrum for this model which consists of a continuum of two-defect scattering states with a lower boundary $\epsilon(q)=J_{1}\left(\frac{5}{4}-|\cos q|\right)$ and, for the restricted range $0.36 \pi<q<0.64 \pi$ of wave numbers, a branch of defect bound states, which emerges from the lower continuum boundary and has a smooth maximum reaching up to $\omega / J_{1}=1$ at $q=\pi / 2$.

In this context, our results suggest that for $q$ near zero or $\pi$, the spectral weight of $S_{z z}(q, \omega)$ is distributed over a broad frequency range of two-defect scattering states. As $q$ approaches $\pi / 2$ from either side, the spectral weight is shifted gradually to the two-defect bound state.

Our result for the dynamic structure factor $S_{D D}(q, \omega)$ is plotted in Fig. 2 for the same set of wave numbers. The line shapes and peak positions resemble those shown in Fig. 1 for

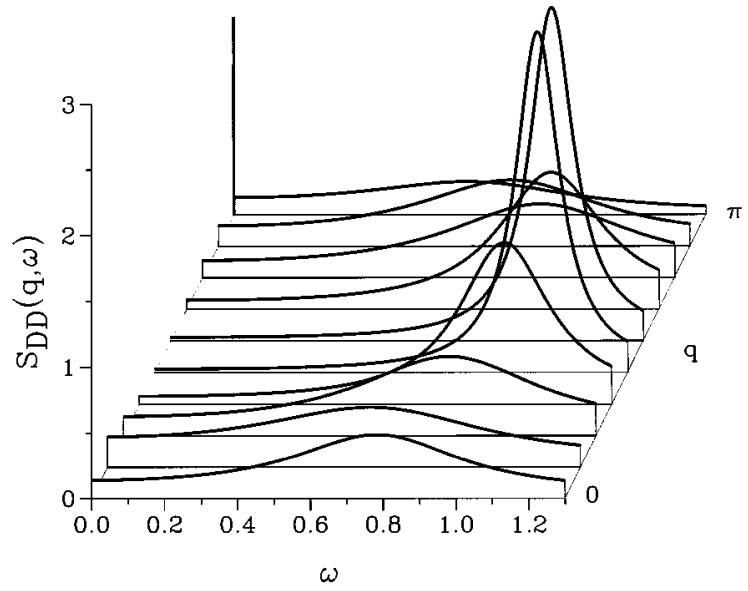

FIG. 2. Dynamic structure factor $S_{D D}(q, \omega)$ vs $\omega$ for $q=2 \pi l / N$, $l=0,1, \ldots, N / 2$ in the dimer ground state $\left|\Phi_{+}\right\rangle$of the Hamiltonian (1) at $J_{2}=0.5$ and $J_{1}=1$ with $N=18$, obtained via strong-coupling continuedfraction reconstruction based on the coefficients $\Delta_{1}, \ldots, \Delta_{9}$ and a Gaussian terminator.

$S_{z z}(q, \omega)$, but there are some notable differences: The spectral weight in $S_{D D}(q, \omega)$ is concentrated at somewhat higher energies. The intensity at $q=0$ is nonzero. The shape of the dimer dispersion is different. $S_{D D}(\pi / 2, \omega)$ does not reduce to a single line. In $S_{D D}(\pi, \omega)$ the spectral weight is shared between a continuum and a $\delta$-peak at $\omega=0$. The latter contribution reflects the presence of dimer long-range order in the ground state.

The Hamiltonian of the 1D $s=1$ model with isotropic bilinear and biquadratic exchange, our second example, is most conveniently expressed in the form

$$
H_{\gamma}=J \sum_{l=1}^{N}\left\{\cos \gamma \mathbf{S}_{l} \cdot \mathbf{S}_{l+1}+\sin \gamma\left(\mathbf{S}_{l} \cdot \mathbf{S}_{l+1}\right)^{2}\right\}
$$

with a single parameter $-\pi<\gamma \leqslant \pi$. More than a decade of research on this model has established a $T=0$ phase diagram consisting of the short-range ordered Haldane phase and three phases with dimer, trimer, and ferromagnetic longrange order. ${ }^{10}$ In the Haldane phase, which includes the Heisenberg antiferromagnet $(\gamma=0)$, the ground state is a non-degenerate singlet state $\left(S_{T}=0\right)$ separated by a gap from the threshold of the excitation spectrum.

At the parameter value $\gamma=\arctan (1 / 3) \simeq 18.4^{\circ}$ within this phase, the ground-state wave function is exactly known. ${ }^{11}$ It is a realization of the so-called valence-bondsolid (VBS) wave function, which can be assembled from the same parts as the dimer state (2). The spin 1 at each lattice site is expressed as a spin-1/2 pair in a triplet state. The singlet-pair forming valence bond involves one fictitious spin $1 / 2$ from each of two neighboring lattice sites. The VBS state can then be regarded as a chain of valence bonds linking successive symmetrized spin-1/2 pairs, which are given by the $S_{T}^{z}=0$ vector of the triplet on each lattice site.

The static spin correlation function in the VBS state, ${ }^{11}$ $\left\langle S_{l}^{z} S_{l+n}^{z}\right\rangle=\frac{4}{3}(-1)^{n} 3^{-|n|},(n \neq 0)$, reflects magnetic shortrange order with a very short correlation length: 


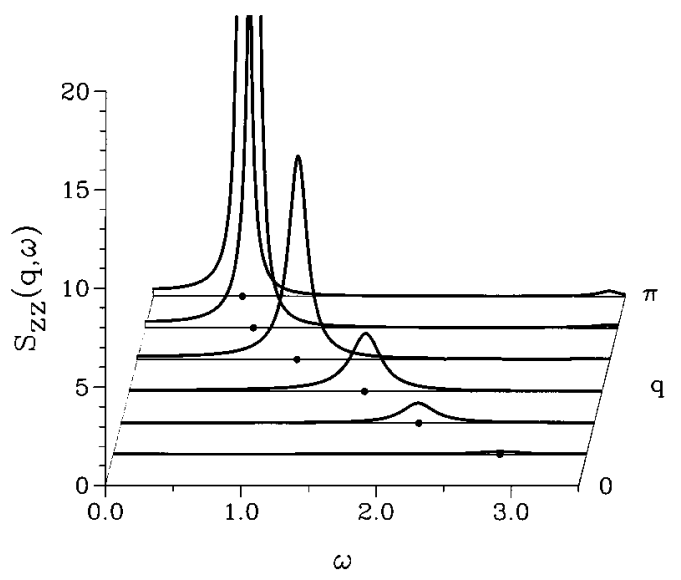

FIG. 3. Dynamic structure factor $S_{z z}(q, \omega)$ vs $\omega$ for $q=2 \pi l / N$, $l=0,1, \ldots, N / 2$ in the VBS ground state of the model system (6) at $J=1$ and $\gamma=\arctan (1 / 3)$ with $N=12$, obtained via a strong-coupling continuedfraction analysis based on the coefficients $\Delta_{1}, \ldots, \Delta_{6}$ and a Gaussian terminator.

$\xi=1 / \ln 3 \simeq 0.91$. The static structure factor is then a nonsingular function with a smooth minimum at $q=0$ and a smooth maximum at $q=\pi$ :

$$
S^{z z}(q)=2(1-\cos q) /(5+3 \cos q) .
$$

The simple structure of the VBS state makes this quantity free of finite-size effects for $q=2 \pi l / N, l=0, \ldots, N-1$. Again, this simplification does not extend to the excitation spectrum and the dynamical properties.

In Fig. 3 we have plotted $S_{z z}(q, \omega)$ as obtained from a strong-coupling continued-fraction analysis with coefficients extracted from the $N=12 \mathrm{VBS}$ wave function. At each value of $q$ the spectral weight of $S_{z z}(q, \omega)$ is found to be concentrated in a single peak with symmetric line shape. The peak frequency decreases monotonically with $q$. The suggested gap value at $q=\pi$ is $\Delta E / J \simeq 0.66$, in good agreement with the result $\Delta E / J \simeq 0.70$ of the single-mode approximation. ${ }^{12}$ The linewidth tends to be very small at $q$ near $\pi$, where the peak frequency is lowest and the intensity highest. It gains considerably in breadth at $q$ near 0 , where the peak frequency is higher and the intensity much lower.

The monotonic $q$-dependence of the dynamically relevant dispersion for $S_{z z}(q, \omega)$ in the VBS state $\left(\gamma \simeq 18.4^{\circ}\right)$ is markedly different from the corresponding quantity in the Heisenberg antiferromagnet $(\gamma=0)$, which belongs to the same phase. In the Heisenberg case, the dispersion has a smooth maximum at $q \simeq \pi / 2$ and smooth minima of unequal height at $q=0$ and $q=\pi .^{13}$

This work was supported by NSF Grant DMR-93-12252 and by the NCSA at Urbana-Champaign.

${ }^{1}$ R. Haydock, Solid State Phys. 35, 215 (1980); M. H. Lee, Phys. Rev. B 26, 2547 (1982); E. R. Gagliano and C. A. Balseiro, ibid. 38, 11766 (1988).

${ }^{2}$ V. S. Viswanath and G. Müller, The Recursion Method. Application to Many-body Dynamics, Lecture Notes in Physics Vol. 23 (Springer, New York, 1994).

${ }^{3}$ V. S. Viswanath, J. Stolze, and G. Müller, J. Appl. Phys. 75, 6057 (1994).

${ }^{4}$ F. D. M. Haldane, Phys. Rev. B 25, 4925 (1982); T. Tonegawa and I. Harada, J. Phys. Soc. Jpn. 56, 2153 (1987).

${ }^{5}$ C. K. Majumdar and D. K. Ghosh, J. Math. Phys. 10, 1388 (1969); P. M. Van den Broek, Phys. Lett. A 77, 261 (1980).

${ }^{6}$ B. S. Shastry and B. Sutherland, Phys. Rev. Lett. 47, 964 (1981).

${ }^{7}$ V. S. Viswanath, S. Zhang, J. Stolze, and G. Müller, Phys. Rev. B 49, 9702 (1994).

${ }^{8}$ W. J. Caspers and W. Magnus, Phys. Lett. A 88, 103 (1982).

${ }^{9}$ The single-mode spectrum of $S_{z z}(\pi / 2, \omega)$ causes the recursion algorithm to terminate spontaneously during the first iteration.

${ }^{10} \mathrm{~A}$ concise recent review was compiled by U. Schollwöck, Th. Jolicoeur, and T. Gorel, Phys. Rev. B (to be published).

${ }^{11}$ I. Affleck, T. Kennedy, E. H. Lieb, and H. Tasaki, Phys. Rev. Lett. 59, 799 (1987).

${ }^{12}$ D. P. Arovas, A. Auerbach, and F. D. M. Haldane, Phys. Rev. Lett. 60, 531 (1988).

${ }^{13}$ O. Golinelli, Th. Jolicoeur, and R. Lacaze, J. Phys. C: Cond. Matter 5, 1399 (1993); S. Haas, J. Riera, and E. Dagotto, Phys. Rev. B 48, 3281 (1993). 